\title{
EchoGéo
}

\section{Penser la ville et l'urbain, les paradoxes de la géographie française}

\section{Anne Hertzog et Alexis Sierra}

\section{(2) OpenEdition}

\section{Journals}

Édition électronique

URL : https://journals.openedition.org/echogeo/11898

DOI : $10.4000 /$ echogeo. 11898

ISSN : 1963-1197

\section{Éditeur}

Pôle de recherche pour l'organisation et la diffusion de l'information géographique (CNRS UMR 8586)

Référence électronique

Anne Hertzog et Alexis Sierra, "Penser la ville et l'urbain, les paradoxes de la géographie française », EchoGéo [En ligne], 12 | 2010, mis en ligne le 31 mai 2010, consulté le 31 juillet 2021. URL : http:// journals.openedition.org/echogeo/11898; DOI : https://doi.org/10.4000/echogeo.11898

Ce document a été généré automatiquement le 31 juillet 2021.

EchoGéo est mis à disposition selon les termes de la licence Creative Commons Attribution - Pas d'Utilisation Commerciale - Pas de Modification 4.0 International (CC BY-NC-ND) 


\title{
Penser la ville et l'urbain, les paradoxes de la géographie française
}

\author{
Anne Hertzog et Alexis Sierra
}

\section{NOTE DE L'AUTEUR}

Cette contribution tente un panorama de la géographie urbaine française afin de mieux éclairer le témoignage des acteurs qui travaillent aujourd'hui sur l'urbain à l'échelle internationale en particulier ceux participant aux événements du type Forum Urbain Mondial. Il ne prétend donc pas être exhaustif.

1 La ville, l'urbain sont des notions qui renvoient à des lieux et à des aires, à des sociétés inscrites territorialement, à des formes spatiales, à des paysages, à des polarisations et à des distances particulières. Aussi, la ville et l'urbain constituent-ils logiquement des objets de la géographie. L'expression " géographie urbaine » utilisée par Jean Bruhnes en 1900 est ainsi antérieure à celui de «sociologie urbaine» (1930) voire de « géographie rurale» (1960). La géographie urbaine, champ de recherche précoce en France est pourtant restée secondaire durant longtemps. Pourtant aussi, les géographes bien qu'ils soient présents dans de nombreux bureaux d'études et agences d'urbanisme le sont beaucoup moins dans les institutions et organismes français qui doivent travailler internationalement sur la ville comme le montrent les témoignages de Yves Dauge et Serge Allou.

2 Dès le début du XXe , Elisée Reclus se passionne pour certaines villes comme Londres et Raoul Blanchard publie en 1911 une étude de géographie urbaine sur Grenoble. Cependant, ce sont davantage des exceptions que la norme : il existe un contraste très fort entre le processus d'urbanisation et le faible intérêt des géographes français pour la ville avant les années cinquante. L’Ecole française de géographie, classique, a fait le choix épistémologique de travailler sur la campagne et le monde rural qui occupe la 
majorité de l'espace. Le paradigme géographique de l'étude de la relation hommemilieu poussait à ne pas travailler sur des terrains urbains. La primauté de la géomorphologie y compris sur le plan institutionnel n'avantageait pas les études urbaines. De ce fait, la géographie urbaine classique française est restée relativement indifférente à certaines orientations ou avancées de la géographie urbaine étrangère. Les approches statistiques restent ignorées en France jusque dans les années soixante. Les approches spatialistes et modélisatrices développées par les sociologues de l'Ecole de Chicago dans les années 30 n'influencèrent que bien plus tard la géographie française. La ville n'y est cependant pas absente. Marie-Claire Robic (2003) relève la précocité et la grande variété des approches de la ville en géographie classique mais de manière fortement segmentée: en témoignent l'approche écologique et monographique de Raoul Blanchard ; l'approche par la morphologie et les paysages de Jean Bruhnes qui utilise une abondante iconographie; ou encore l'approche régionale et plutôt économiste, avec la description des fonctions urbaines et des relations ville/ campagne, dès Vidal de la Blache et plus fortement chez ses successeurs dans les années trente.

3 Après une période d'affirmation foisonnante des études urbaines durant l'entre-deux guerre, le tournant majeur est opéré dans les années 50-60. Paul Claval montre que cela tient à la fois tant au renouvellement d'ensemble de la géographie qu'au contexte politique et social : l'aménagement du territoire à la suite de la reconstruction et en pleine expansion économique influe fortement les choix et les carrières des géographes. L'accent est en particulier mis sur les fonctions urbaines, sur le cadre matériel de la ville, sur la démographie comme en témoigne le Traité de Géographie urbaine de George Chabot et Jacqueline Beaujeu-Garnier important ouvrage de synthèse publié en 1964. L'inquiétude sur la maîtrise de la croissance urbaine et sur les fonctions d'encadrement territorial oriente certaines études. Mais Pierre George ou Michel Rochefort portent l'idée que la modernisation du pays passe par les villes, promeuvent une implication des géographes dans l'action, pensent l'aménagement du territoire qui passe alors par l'organisation de réseaux urbains. Une approche synthétique de la ville apparaît alors durant ces Trente Glorieuses y compris sur les villes étrangères (George, 1952). Les institutions nationales comme la DATAR recrutent les géographes, s'en inspirent et en retour orientent les recherches. Elles furent relayées à partir des années quatre-vingt par les agences et instituts d'aménagement et d'urbanisme tant au niveau municipal que régional (voir à ce sujet le dossier Sur le métier, Echogéo, $n^{\circ} 11$ http:// echogeo.revues.org/index11718.html )

4 Pour autant, la difficulté restait encore grande de proposer une définition de la ville, "objet spatial complexe et multidimensionnel», donc scientifiquement légitime (Lussault, Lévy, 2003). De même, la logique institutionnelle, héritée de la colonisation, ne pousse pas les géographes à travailler sur les villes du Tiers-monde. La coopération universitaire ou pour le développement s'oriente prioritairement vers les études agricoles et rurales.

5 A partir des travaux anglo-saxons des années 40 à 60 et du développement des outils informatiques, la géographie urbaine se renouvelle à travers les approches de la ville conçue comme un objet inscrit dans un espace qu'il polarise et intégré dans un système. La ville est étudiée «non comme entité isolée mais comme élément d'un territoire ou d'un système de villes » (Pumain et alii, 1996). Cela conduit à l'analyse de l'urbanisation et de ses processus, non plus ville par ville, mais dans une étude globale qui conclue sur 
la concentration généralisée de la population à l'échelle planétaire. Ces recherches se prolongent dans les années 90 par des études qui considèrent la ville comme un nœud dans un réseau de relations. L'objectif est de comprendre les interdépendances entre les villes, l'analyse des hiérarchies urbaines décryptées à l'aide d'indicateurs synthétiques et la capacité des pôles urbains à établir des connexions et à exploiter des situations. Cette géographie insistant sur les systèmes urbains fait écho au développement du ranking dans les institutions internationales, nationales et locales pour positionner les villes dans un contexte international interdépendant.

Le processus de mondialisation influence alors la géographie urbaine notamment à partir des travaux de Olivier Dollfus qui théorise l'Archipel Mégalopolitain Mondial au milieu des années 1990, comme «l'ensemble des villes qui contribuent à la direction du monde » et donc en valorisant le rôle croissant des relations intermétropolitaines. C'est précisément durant la décennie 1990 que naissent les conférences onusiennes sur la ville à partir de la Conférence Habitat II de Istanbul (1996). Aux échelles européenne et nationale, l'intérêt pour les réseaux de métropoles régionales et nationales s'accroît dans un contexte d'ouverture des frontières. Les politiques nationales mettent parallèlement l'accent sur une concentration de difficultés sociales dans certains espaces urbains dont la mise en place de politiques de la ville est l'expression.

6 L'émergence des géographies sociale, culturelle et politique a entraîné un profond renouvellement des approches des espaces urbains à partir des années 1970-80. Les études internes centrent l'analyse sur les sociétés urbaines. L'objectif prioritaire est de comprendre l'organisation intra-urbaine au double sens du mot organisation: ce qui organise (les acteurs et leurs logiques), ce qui est organisé (formes, paysages, types d'espaces). La notion de "production de l'espace » oriente dans un premier temps les études sur des espaces urbains socialement différenciés avec parfois des visées modélisatrices (centre/périphérie). Cela conduit à s'intéresser à la distribution de la population, à analyser les thèmes de la ségrégation, des mobilités et des recompositions socio-spatiales. Les recherches consacrées aux politiques publiques et à la gestion urbaine se développent avec un intérêt de plus en plus fréquent pour les représentations socio-spatiales de la ville et les pratiques de ses habitants. La dimension physique ne disparaît pas : la ville est considérée comme un environnement construit qui permet de renouveler la question de la relation homme/milieu. La problématique des risques d'origine naturelle ou technologique interroge la ville à la fois comme système vulnérable et comme foyer d'aléas. L'espace urbain devient d'autant plus complexe à gérer en situation d'incertitude que la ville concentre les enjeux.

7 Avec le développement des mobilités et de nouvelles pratiques, avec également les dynamiques à l'œuvre sur l'ensemble du monde, les notions de ville et d'urbain se différencient. Si les conférences et fora urbains tentent de penser la ville de demain, il y a bien la "disparition d'une certaine manière locale de vivre institutionnellement ensemble, qui fut le propre des entités dotées d'une identité et qu'on appelait ville » (Choay, 1999). Les outils de la géographie classique, trop souvent persistants, deviennent inopérants pour comprendre les phénomènes liés à la ville. Au cours des années 1990, Jacques Lévy propose une théorie de l'urbain comme organisation spatiale caractérisée par un jeu de couple entre densité et diversité, l'urbain étant toujours plus dense et plus divers que le non urbain. Des degrés d'urbanité sont alors définis qui permettent de questionner l'urbain et ses dynamiques dans le contexte de la mondialisation (1999). Les questions de l'étalement urbain (périurbanisation) et de 
l'organisation réticulaire des espaces urbains alimentent les réflexions sur la « fin de la ville " ou la "ville émergente" (Chalas, Dubois-Taine, 1996) et conduisent à des redéfinitions comme l'exprime la «métapole» de François Ascher (1995). La question de la fragmentation urbaine devient fondamentale et imprègne le questionnement sur la gouvernance urbaine. Elle interroge sur ce qui «fait ville» et ce qu'est être urbain : les pratiques, les représentations, les images participent à une géographie des identités urbaines et de la citadinité (Gervais-Lambony, 2007). Le développement généralisé des mobilités (migratoires, touristiques...) contribue à redéfinir les territorialités urbaines (Capron G., Cortes G., Guétat-Bernard H.,2005,) et l'urbanité (Knafou, Duhamel, 2007).

En portant les thèmes du développement durable, de la gouvernance, des discriminations et du genre, de la gestion dans l'incertitude voire la question climatiques, les instances internationales mettent en exergue la ville et la société urbaine à la fois comme lieu d'identification des problèmes et comme laboratoire de nouvelles politiques. La question urbaine est porteuse de transversalité comme le montrent les conférences internationales à travers des notions-slogans comme ceux de la ville durable, la ville citoyenne ou la ville sûre. Tous les champs disciplinaires sont alors sollicités mais la géographie peine encore à se situer entre les préoccupations sociales et l'implication à laquelle elles peuvent conduire, et une recherche scientifique qui reste critique envers la terminologie utilisée ${ }^{1}$ et qui doit intégrer la diversité (et la relativité) des points de vue.

Ces différents points de vue sur la ville et l'urbain se déclinent enfin dans les différentes aires régionales. Si la mondialisation comme affirmation d'un niveau d'analyse pertinent est progressivement intégré aux études urbaines, la distinction Nord/Sud tout comme les spécialisations régionales persistent. La première est fortement influencée par les paradigmes passés (géographie tropicale puis du développement) ainsi que par les institutions et les politiques qui distinguent les actions menées au Sud de celles conduites en Amérique du Nord, en Europe ou au Japon. Malgré la similarité des dynamiques qui traversent le monde, malgré l'imbrication d'espaces urbains de très inégal développement aussi bien dans les métropoles nord-américaines et européennes qu'en Amérique latine, en Afrique ou en Asie, cette institutionnalisation explique en partie la distinction Nord/Sud des études urbaines. Les études comparatives se multiplient cependant. Elles se développent d'une part, entre régions du monde comme le montre le programme ANR Périsud ${ }^{2}$ et d'autre part, entre " Nords » et «Suds », comme en témoignent les recherches menées au sein du laboratoire Gecko ${ }^{3}$.

Bibliographie

\section{BIBLIOGRAPHIE}

AscherF., 1995. Métapolis ou l'avenir des villes. Odile Jacob. 
Capron G., Cortes G., Guétat-Bernard H., 2005. Liens et lieux de la mobilité. Ces autres territoires. Belin.

Chabot G., Beaujeu-Garnier J., 1964. Traité de géographie urbaine. Colin.

Choay F., 1999. De la ville à l'urbain. Urbanisme, n³09.

Derycke P-H., Huriot J-M., Pumain D., 1996. Penser la ville. Anthropos, collection Villes.

Dollfus O., 1997. La mondialisation. Presses de Sciences Po, collection La bibliothèque du citoyen.

Dubois-Taine G., Chalas Y., 1998. La ville émergente. Editions de l'Aube.

George P., 1952. La ville, le fait urbain à travers le monde. PUF.

Gervais-Lambony P., Dorier Apprill E . (dir.), 2007. Vies citadines. Belin.

Lévy J., 1999. Le tournant géographique. Belin.

Lefort I., 2008. Deux méta-discours et leurs usages géographiques : mondialisation et

développement durable ; Bulletin de l'Association de Géographes Français, 2008-3, p. 361-369

Lussault M., 2000. La ville des géographes. In Body-Gendrot S., Lussault M, Paquot T., La Ville et

l'urbain, l'état des savoirs, La Découverte, p. 21-34.

Lussault M., 2003. Géographie urbaine. Dictionnaire de la géographie et de l'espace des sociétés, Belin.

Knafou R., Duhamel P., 2007. Mondes urbains du tourisme. Belin.

Robic M-C., 2003. La géographie urbaine en France, 1890-1960. Sociétés contemporaines, $\mathrm{n}^{\circ}$ 49-50, $\mathrm{p}$. 107-138.

\section{NOTES}

1. Voir à ce sujet la réflexion développée par Isabelle Lefort sur le développement durable et la mondialisation (Lefort, 2008)

2. Programme de recherche sur le périurbain dont les terrains sont Mexico, Lima, Abidjan, Le Cap, Shanghaï, Hanoï

3. Laboratoire de géographie comparée des Nords et des Suds, université de Paris X

\section{AUTEURS}

\section{ANNE HERTZOG}

Anne Hertzog est maître de conférences en géographie à l'Université de Cergy-Pontoise. Elle est rattachée au laboratoire MRTE de l'UCP et associée à l'EIREST(université Paris 1)

hertzog.anne@wanadoo.fr

\section{ALEXIS SIERRA}

Alexis Sierra est maître de conférences, géographe et membre de l'UMR PRODIG alexisierra2001@yahoo.fr 